\title{
Future Feasibility of Using Wearable Interfaces to Provide Social Support
}

\author{
Natalie Wilde*, Hamed Haddadi* ${ }^{*}$, Akram Alomainy* \\ *Queen Mary University of London, UK \\ ${ }^{\dagger}$ Qatar Computing Research Institute, Qatar \\ n.wilde@qmul.ac.uk
}

\begin{abstract}
Social support has a positive influence on a person's overall wellbeing. The recent creation of mobile and online social networks have changed the methods used to obtain such support. Previous wearable devices have focussed on increasing an individuals perceived level of social support by either encouraging new social relationships or strengthening those already existing. With the release of the Apple Watch, wearable interfaces are becoming popular but there is little research into the current attitudes of using these interfaces as a social support medium. In this paper we present the results of our survey to establish the attitudes of current smartwatch owners. Results show owning a smartwatch has no effect on the levels of social support a person feels they have. The most commonly used method of support was through smartphone, which was true for people regardless of whether they own a smartwatch. A large number of smartwatch owners stated using their device was their last preference in seeking emotional (61\%) and informational (57\%) support from others. Results from the survey indicate that more research is needed to establish exactly what factors make technological devices well suited to accommodate social support and how these can be applied to wearable interfaces in the future.
\end{abstract}

\section{Categories and Subject Descriptors}

H.5.m [Information Interfaces And Presentation]: Miscellaneous; J.3 [Computer Applications]: Life And Medical Sciences-Consumer Health

\section{General Terms}

Social Support, Wearable Technology, Social Relationships

\section{Keywords}

Smart Watch, Wearable Interfaces, Social Networks

\section{INTRODUCTION}

Social support can be described as any type of communication, both verbal and non verbal, that reduces an individuals uncertainty. It helps an individual feel as if they have increased control of either themselves or the situation that is causing them distress [1]. Long term social support is usually provided by the individual's informal network, which includes their family and friends. But support can also be provided from more formal networks such as their doctor or a councillor.

Social support offers many benefits to an individuals overall wellbeing, regardless of stress levels [3]. This is because social support meets basic human needs for a sense of belonging and reassurance of one's self worth. Having adequate social support available ensures that stressful events are handled and coped with in a way that minimises the negative effects on one's health. This is called the Buffering Effect and helps to keep both short and long term health consequences low [11].

There are two distinct measurements of social support. Actual support is the amount of support that is given to the individual, either in what others have said or done for them. But another measurement which is proven to be of greater importance is that of perceived social support; the amount of support that the individual feels is available to them. In previous studies, it has been found that perceived social support is what actually contributes to good health and wellbeing within an individual [17]. This shows the perception of support to be subjective, what works for one person may not be perceived so beneficial by the next.

An individual obtains social support by accessing and utilising their social networks. Previous research has stated that offline social networks can have a positive effect on a person's wellbeing [8]. Recent advances in technology have changed the whole structure of social networks and how they are accessed. The development of internet based support groups and online social networks (OSN) have recently gained in popularity [9]. These online virtual communities come together to share common interests, experiences and to offer support to each other. OSN offer benefits for an individual as they can seek support at any time and from any geographical location. There are also mobile social networks (MSN) that allow groups of people to be accessed and engaged with from one's mobile device [4]. Previous studies have found MSN to be beneficial in offering social support 
in both verbal and non verbal communication $[6,13]$.

As wearables become more widely available to the consumer, this may give rise to a new type of wearable social network. There is little work within this area and how wearable technology is perceived to affect levels of social support in the user. With the recent release of the apple watch, this paper aims to highlight the current attitudes of smart watch owners with using a wearable interface for seeking social support. Opinions are collected in the form of an online survey. The survey focusses on two main types of social support; emotional and informational. Emotional support includes needing reassurance, affection and someone to show concern about specific issues. Informational support includes advice, guidance, suggestions and useful information about an issue. The rest of the paper is organised as follows. The current methods used by wearable technologies to aid social support are discussed. The methodology behind the survey and analysis are outlined. Then the results of the survey are presented and discussed to offer directions for future work.

\section{BACKGROUND}

Currently there are two methods used by wearables to strengthen the users perceived level of social support. The first is to help the user create new social relationships and links within their social network. The more people they have in their network, the more likely they are to have someone they can turn to in times of needing support. The Memetag device is worn around the users neck and allows users to share their ideas and opinions with each other [4]. The tag consists of a LCD Screen with red and green buttons for accepting and deleting memes. Users wearing the same tag can like each others memes when they meet. These small devices are all connected to community mirrors. These are large public displays that show real time visualisations of the community dynamics. From looking at the community mirror, an individual may be able to pick out people they feel they want to form relationships with. Studies held at a conference found the device to be effective at supporting the users in the formative stages of social network building.

More recently in [10], Kan et al. developed a t-shirt that also aims to make it easier to form new social relationships. The t-shirt has letters on the front printed with thermochromic ink, which is coloured ink that turns transparent at $89^{\circ} \mathrm{F}$. When two users wearing the t-shirt high five, certain words are highlighted. These words reveal common interests between the wearers and aim to serve as a social catalyst. In [7], Chambers et al. focussed on easing the problem of social isolation. They developed a wearable application that used play as a method to increase an individuals levels of social support. It did this by awarding badges and points every time the user carried out social gestures on others, such as shaking hands.

The second method used to increase perceive social support is to enhance the social relationships that an individual already has. One way to achieve this is by facilitating social support between people over great distances. Technologies developed have allowed people in two different geographical locations to support each others running sessions [12].

Devices have also started to use touch to strengthen relationships between users. Previous studies have shown touch to play an important role in interpersonal communications and therefore the maintenance of social support networks [16]. Devices created such as the Smartstones touch ${ }^{1}$ allow communication through touch and gestures to be sent in the form of vibrations.

Sociometric Badges are wearable devices that automatically track the wearers face to face interactions and conversational times [14]. They achieve this through analysing social signals obtained from vocal features, relative location and the wearers body motion. This data can then be presented back to the wearer for reflection and to provide support on their social behaviour. Examples of sociometric badge uses include conferences $[4,5]$ and analysing childrens social behaviour in kindergarden [15].

\section{SURVEY METHODS}

The study conducted aimed to highlight smartwatch owners thoughts towards using wearable interfaces to obtain social support. Participants to the survey were recruited through the OSN platforms Reddit and Facebook. On Reddit, online communities based around smartwatches such as the Pebble, Moto 360 and Apple Watch were selected as potential participants. The survey itself was created and shared through the google forms platform for ease of distribution across the internet via online forums.

The survey questionnaire contained 17 questions split into three parts; A, B and C. Before the survey each participant was presented with an information page and consent form to sign. Part A of the questionnaire asked the participant about the technological devices that they own and their current social support habits. The final question of part A asked the participant if they ever ask for help and advice from others. If the Participant responded to this with a 'yes' they were required to fill out both parts $\mathrm{B}$ and $\mathrm{C}$. If they answered 'no' only Part $\mathrm{C}$ was required. Before part $\mathrm{B}$, the participants were given a definition of social support to aid them in answering the survey. Part $B$ of the survey asked more in depth questions regarding the participants preferences in ways of receiving social support. Respondents where given a list of mediums to rate in order of preference for receiving different types of social support. This list included face to face, desktop computer, laptop, tablet, smartphone and smartwatch. The types of social support questioned were emotional and informational types of social support. Before answering the questions, a definition and example of each type of social support was explained to the participant. It also asked how easy they find obtaining social support through their smartwatch devices. Finally, part $\mathrm{C}$ of the survey contained a set of demographics questions.

\section{RESULTS}

A total of 266 respondents completed the survey. Of these, $177(66.5 \%)$ already owned a smartwatch device. When studying the demographics of smartwatch owners there were a couple of observations. A high majority of smartwatch owners were male $(87.5 \%)$, which was found to be statistically significant $(\mathrm{p}<.001)$. The age group of smartwatch

\footnotetext{
$\overline{{ }^{1} \mathrm{http}: / / \text { www.smartstones.co/ }}$
} 


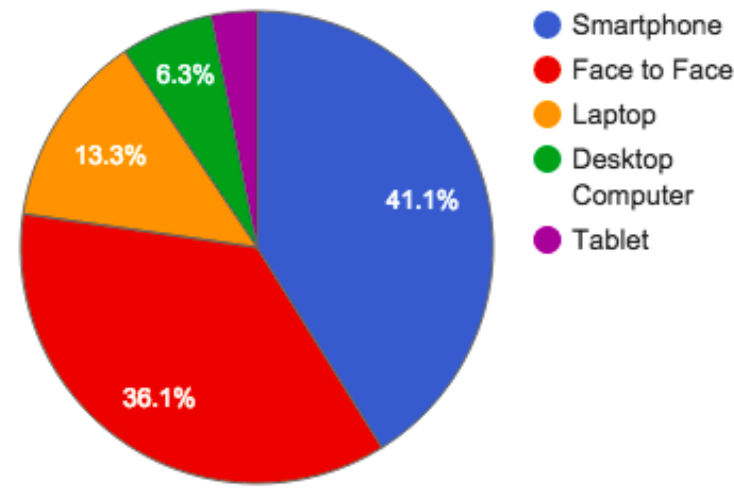

Figure 1: Method of communication currently used for Social Support

owners was also found to be significant ( $<$.001), rejecting a null hypothesis that age and gender of smartwatch owners is equally distributed. Of smartwatch owners, 96 (54.2\%) were aged 18 - 25 and there were a further 59 (33.3\%) within the 26 - 35 category. 258 (97\%) of the respondents state that they use technology to communicate with friends and family at least every day.

For the next section, only the data from respondents who owned smartwatches was analysed. 126 (77.3\%) respondents agreed with the statement 'Social support is very important to my overall happiness and wellbeing'. This was found to reject a null hypothesis that social support has no effect on happiness levels $(\mathrm{p}<.001)$. When asked if they agreed with the statement 'I am fully socially supported', 105 respondents $(70.5 \%)$ answered 'yes'. When comparing data between groups, there was no significant difference between owning a smartwatch and not. This means that the owning of a smartwatch has no effect on perceived levels of social support. 109 (61.6\%) respondents stated that they do seek support and advice from others so were able to continue to complete Part B of the survey.

When asked what method of communication they use to talk to family and friends, 48 respondents (44\%) stated their smartphone, making it the most common method as shown in Figure 1. Significantly, no one stated their smartwatch as a preferred communication device $(\mathrm{p}<0.01)$. At this stage, data from smartwatch owners and non smartwatch owners were compared. When comparing commonly used devices for obtaining social support, the smartwatch option was omitted from the significance test to eliminate bias. No difference was found between the two groups most commonly used method for receiving social support.

When the smartwatch owners were asked to rank technological devices in order of preference for receiving emotional and informational support, Figure 2 shows the results. For emotional support the smartwatch was not anyone's first or second preference. A majority of respondents $(61 \%)$ stated it as their last preference in seeking emotional support from others. Preferences were similar for informational support also, with $59(57 \%)$ of respondents placing

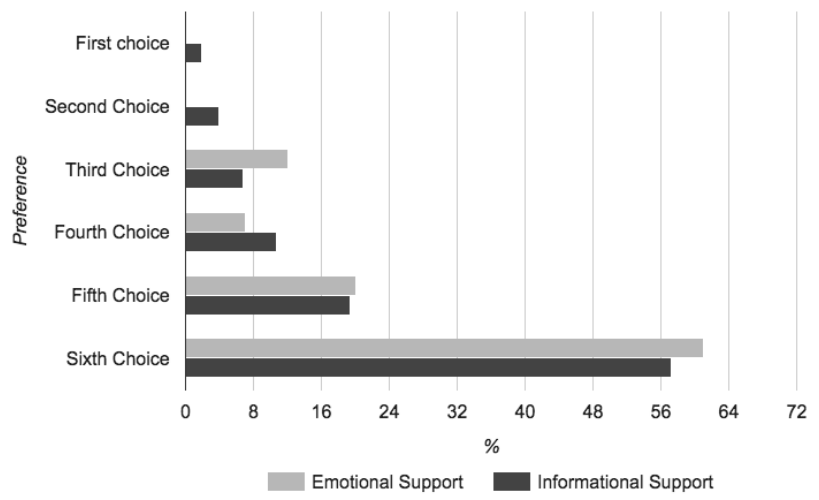

Figure 2: Preference of using smartwatch for support over other mediums

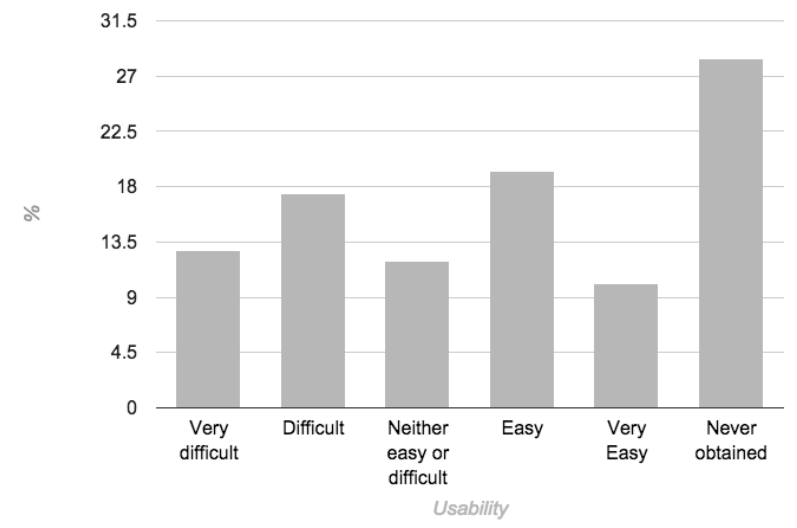

Figure 3: Ease of use of smartwatch in obtaining social support

smartwatches in last place. These rankings of preference for both emotional and informational support were found to be statistically significant $(\mathrm{p}<.001)$.

When asked how easy they found obtaining social support through their smartwatch, there were mixed responses as shown in Figure 3. Roughly equal numbers of respondents found smartwatches both difficult and easy to use for social support. 31 (28.4\%) respondents had never used their smartwatch for obtaining social support. There was no difference with regards to gender or age as perceived ease of use of a smartwatch was fairly evenly distributed.

\section{DISCUSSION AND FUTURE WORK}

When referring to the technology adoption lifecycle [2], smartwatches appear to be in the early adopters stage. When looking into the demographics of people who make up these early adopters, the majority are male and aged between 18 - 25. This could indicate the current average user profile, indicating that wearable smartwatches are not appealing to the female consumer at the current moment. This could be because of aesthetics or function. A large amount of respondents use technology to communicate with family and friends and obtain social support currently. 
A majority of smartwatch owners agreed that social support is important for their happiness which suggests that they would prefer social support on a regular basis. $70.5 \%$ of owners felt that they were fully socially supported but this is no different to the proportion when looking at people that do not own smartwatches. This could suggest that wearable technology is not enhancing social support levels at the current time. This could be down to the fact that the technology is still relatively new, there are not many current applications that deal with social support specifically. It may also be down to the fact that the user is getting support, but the wrong kind for what they require at the time. For example they may be getting informational support when they desire emotional. Also applications that allow support are still in the early stages development wise and they are improving their designs on a daily basis. Another possibility could be that the watch interface and form itself is just not effective in raising the levels of social support a person feels that they have. The fact that $61.6 \%$ say that they actively seek social support would suggest the need for more research into wearable interfaces that can accommodate these needs.

The fact that nobody chose their smartwatch as their most commonly used device for social support could be down to it been a relatively new technology. But observing that there is no difference in usage regardless of whether they own a smartwatch suggests that it does not offer the needed ease and functionality that other devices such as the smartphone do. Future work could focus on the exact reasons why people prefer certain mediums and use this to build a stronger application for the smartwatch. For example, do people prefer texting or talking?

Smartwatch owners also do not prefer to use their smartwatch for seeking emotional or informational support from others. This further backs up that the current interfaces used are not suitable. Future work could focus on why it is not working for them and generate systems that encourage the use of wearable interfaces for social support. There was no difference between type of support and preference, which suggests future work should look into how the two types of support differ from each other and the best way support can be given through wearable devices.

There are a large number of smartwatch users that are not using their device at all for seeking social support. This is a statement that needs questioning further. Is it because of their personal attitudes towards the device or because the device does not work for social support at the current time? Users are finding the interface both difficult and easy to use, suggesting that there is no current system designed for seeking social support through these devices. Research into designing an interface that makes it easy for everyone could be beneficial for future developers.

Overall preference is towards smartphones when it comes to seeking social support, possibly due to portability. Over the years devices have got smaller and more ubiquitous; fitting around peoples lifestyles and social networks. It would appear that the smartwatch is not building on the smartphones previous success $[13,6]$. Much more research into the future interface design of these devices is needed to encourage the use of wearable devices for social support.

\section{ACKNOWLEDGMENTS}

The authors would like to thank all the people that participated in the survey questionnaire. This work is supported with funding from Engineering and Physical Sciences Research Council (EPSRC) and the Arts and Humanities Research Council (AHRC).

\section{REFERENCES}

[1] T. L. Albrecht and M. B. Adelman. Communicating social support. Sage Publications, Inc, 1987.

[2] G. M. Beal, J. M. Bohlen, et al. The diffusion process. Agricultural Experiment Station, Iowa State College, 1957.

[3] L. F. Berkman and T. Glass. Social integration, social networks, social support, and health. Social epidemiology, 1:137-173, 2000.

[4] R. Borovoy, F. Martin, S. Vemuri, M. Resnick, B. Silverman, and C. Hancock. Meme tags and community mirrors: moving from conferences to collaboration. In Proceedings of the 1998 ACM conference on Computer supported cooperative work, pages 159-168. ACM, 1998.

[5] R. Borovoy, M. McDonald, F. Martin, and M. Resnick. Things that blink: Computationally augmented name tags. IBM Systems Journal, 35(3.4):488-495, 1996.

[6] L. M. Brown and J. Williamson. Shake2talk: multimodal messaging for interpersonal communication. In Haptic and Audio Interaction Design, pages 44-55. Springer, 2007.

[7] T. Chambers. Promoting social interactions through a wearable computing application.

[8] M. Greenblatt, R. M. Becerra, and E. Serafetinides. Social networks and mental health: An overview. The American journal of psychiatry, 1982.

[9] C. A. Heaney and B. A. Israel. Social networks and social support. Health behavior and health education: Theory, research, and practice, 4:189-210, 2008.

[10] V. Kan, K. Fujii, J. Amores, C. L. Zhu Jin, P. Maes, and H. Ishii. Social textiles: Social affordances and icebreaking interactions through wearable social messaging. In Proceedings of the Ninth International Conference on Tangible, Embedded, and Embodied Interaction, pages 619-624. ACM, 2015.

[11] M. Mattson and J. G. Hall. Health as communication nexus: A service-learning approach. 2011.

[12] F. Mueller, S. O'Brien, and A. Thorogood. Jogging over a distance: supporting a jogging together experience although being apart. In CHI'07 extended abstracts on Human factors in computing systems, pages 1989-1994. ACM, 2007.

[13] A. U. Mutsuddi and K. Connelly. Text messages for encouraging physical activity are they effective after the novelty effect wears off? In Pervasive Computing Technologies for Healthcare (PervasiveHealth), 2012 6th International Conference on, pages 33-40. IEEE, 2012.

[14] D. O. Olguin and A. S. Pentland. Sociometric badges: State of the art and future applications. 2007.

[15] S. Park, I. Locher, A. Savvides, M. B. Srivastava, A. Chen, R. Muntz, and S. Yuen. Design of a wearable sensor badge for smart kindergarten. In Wearable Computers, 2002.(ISWC 2002). Proceedings. Sixth International Symposium on, pages 231-238. IEEE, 2002.

[16] R. Wang, F. Quek, J. K. Teh, A. D. Cheok, and S. R. Lai. Design and evaluation of a wearable remote social touch device. In International Conference on Multimodal Interfaces and the Workshop on Machine Learning for Multimodal Interaction, page 45. ACM, 2010.

[17] E. Wethington and R. C. Kessler. Perceived support, received support, and adjustment to stressful life events. Journal of Health and Social behavior, pages 78-89, 1986. 